ANNALES

POLONICI MATHEMATICI

$85.2(2005)$

\title{
Kamenev type oscillation criteria for second order matrix differential systems with damping
}

\author{
by QI-Gui YAng (Guangzhou) and Sui Sun Cheng (Hsinchu)
}

\begin{abstract}
By using monotone functionals and positive linear functionals on a suitable matrix space, new oscillation criteria for second order self-adjoint matrix differential systems with damping are given. The results are extensions of the Kamenev type oscillation criteria obtained by Wong for second order self-adjoint matrix differential systems with damping. These extensions also include an earlier result of Erbe et al.
\end{abstract}

1. Introduction. Oscillations of mechanical systems with damping are often described by second order matrix differential equations of the form

$$
\left(P(t) Y^{\prime}(t)\right)^{\prime}+r(t) P(t) Y^{\prime}(t)+Q(t) Y(t)=0, \quad t \geq t_{0},
$$

where $r(t)$ is a real continuous function on $\left[t_{0}, \infty\right)$ and $Y(t), P(t), Q(t) \in$ $\mathbb{R}^{n \times n}$ are real symmetric continuous matrix functions for $t \in\left[t_{0}, \infty\right)$ with $P(t)$ positive definite. A solution $Y(t)$ of the system (1) is said to be nontrivial if $\operatorname{det} Y(t) \neq 0$ for at least one $t \in\left[t_{0}, \infty\right)$, and prepared or self-conjugate if

$$
Y^{*}(t) P(t) Y^{\prime}(t)-\left[Y^{*}(t)\right]^{\prime} P(t) Y(t)=0, \quad t \geq t_{0},
$$

where $A^{*}$ denotes the transpose of $A$. A prepared solution $Y(t)$ of $(1)$ is called oscillatory on $\left[t_{0}, \infty\right)$ if its determinant vanishes at some point of $[T, \infty)$ for each $T \geq t_{0}$. Otherwise, it is called nonoscillatory. The system (1) is called oscillatory on $\left[t_{0}, \infty\right)$ if every nontrivial prepared solution is oscillatory.

Oscillation criteria for (1) have been given by a number of authors. In particular, Erbe et al. in [1] obtains Kamenev type oscillation condition for (1) with $r(t) \equiv 0$.

On the other hand, when $n=1$ and $P(t) \equiv I$, the system (1) reduces to a scalar differential equation of the form

$$
y^{\prime \prime}+r(t) y^{\prime}+q(t) y=0, \quad t \geq t_{0},
$$

2000 Mathematics Subject Classification: 34A30, 34C10.

Key words and phrases: oscillation, matrix differential system, damping.

Project 10461002 supported by NSFC and by the Science Foundation of Guangxi Province of China. 
investigated by Wong in [3] where nontrivial extensions of the earlier results for the scalar equation are given. However, no further generalizations have been given by Wong for the matrix system (1).

In this paper, by employing the ideas of Wong and by using monotone functionals as well as positive linear functionals, we will obtain three oscillation theorems providing nontrivial extensions of some of the earlier results.

Let $D=\left\{(t, s): t_{0} \leq s \leq t\right\} \subset \mathbb{R}^{2}$ and $D^{0}=\left\{(t, s): t_{0} \leq s<t\right\}$, and let $H(t, s)$ be a kernel function continuous and sufficiently smooth on $D$, so that the following conditions are satisfied:

$$
H(t, s) \geq 0 \text { and } H(t, t)=0 \text { for } t_{0} \leq s \leq t ;
$$

(H2) For each $s \geq t_{0}, \lim _{t \rightarrow \infty} H(t, s)=\infty$, and there exist positive constants $k_{0}, K_{0}$ such that $0<k_{0} \leq \lim _{t \rightarrow \infty} H(t, s) H^{-1}\left(t, t_{0}\right) \leq K_{0}<\infty$ for $s \geq t_{0}$;

$$
0 \leq-\bar{\partial} H(t, s) / \partial s=\lambda(t, s) H(t, s) \text {, and } \partial \lambda(t, s) / \partial s \leq 0 \text { for }(t, s) \in D^{0} .
$$

Furthermore, let $\varrho \in C^{1}\left(\left[t_{0}, \infty\right),(0, \infty)\right)$ and let $\mathbb{K}=C^{1}\left(\left[t_{0}, \infty\right), \mathbb{R}^{n \times n}\right)$. The operator $A_{\tau}^{\varrho}: \mathbb{K} \rightarrow \mathbb{K}$ (see [3]) is defined as

$$
A_{\tau}^{\varrho}(g)(t)=\int_{\tau}^{t} H(t, s) g(s) \varrho(s) d s, \quad t \geq \tau \geq t_{0},
$$

where $g \in C\left[t_{0}, \infty\right)$. As shown in [3], the integral operator $A_{\tau}^{\varrho}$ is linear and positive (see def. below) and has the following properties:

$$
\begin{aligned}
& \lim _{t \rightarrow \infty} \frac{1}{H\left(t, t_{0}\right)} \int_{t_{0}}^{t} H(t, s) g(s) d s=0 \text { if } g \in C\left[t_{0}, \infty\right) \text { and } g \in L^{1}\left[t_{0}, \infty\right) \\
& \lim _{t \rightarrow \infty} \frac{1}{H\left(t, t_{0}\right)} \int_{t_{0}}^{t} H(t, s) g(s) d s=0 \text { if } g \in C\left[t_{0}, \infty\right) \text { and } \\
& \lim _{t \rightarrow \infty} \int_{t_{0}}^{t} g(s) d s=\infty ; \\
& \frac{1}{H\left(t, t_{0}\right)} \int_{t_{0}}^{t} H(t, s) g(s) d s \text { is nondecreasing in } t \text { if } g \in C\left[t_{0}, \infty\right) \text { and } \\
& g(s) \geq 0 \text { on }\left[t_{0}, \infty\right) ; \\
& A_{\tau}^{\varrho}\left(g^{\prime}\right)(t)=-H(t, \tau) g(\tau) \varrho(\tau)+A_{\tau}^{\varrho}\left(\left[\lambda-\varrho^{-1} \varrho^{\prime}\right] g\right)(t) \text { for } g \in C^{1}\left[t_{0}, \infty\right) .
\end{aligned}
$$

2. Oscillation in terms of nonlinear functionals. Hereafter $\operatorname{tr}(A)$ will denote the trace of $A \in \mathbb{R}^{n \times n}$ and $I_{n}$ or $I$ the $n \times n$ identity matrix. Let $\mathcal{S}$ denote the linear space of all $n \times n$ real symmetric matrices. For any $A, B \in \mathcal{S}$, we write $A \geq B$ or $A-B \geq 0$ if $A-B$ is positive semi-definite, and $A>B$ or $A-B>0$ if $A-B$ is positive definite. We will use some 
properties of this ordering, viz. $A \geq B$ implies that $C A C \geq C B C$ for $C \in \mathcal{S}$ and $\operatorname{tr} A \geq \operatorname{tr} B$, and $A \geq B$ and $B \geq 0$ imply that $A \geq 0$.

Definition 2.1. A functional $p: \mathcal{S} \rightarrow \mathbb{R}$ is said to be subhomogeneous if $p(\lambda K) \leq \lambda p(K)$ whenever $K \in \mathcal{S}$ and $\lambda \geq 0$. Such a functional is said to be monotone (or nondecreasing) if $J-K \geq 0$ implies $p(J) \geq p(K)$ for $J, K \in \mathcal{S}$.

The first part of Definition 2.1 is found in Hartman [2, p. 328]. Note that the functionals $p(K)=\lambda_{\max }(K), \lambda_{\max }(K+P), \operatorname{tr}(K+P)$, where $P \in \mathcal{S}$ is positive definite, are monotone and subhomogeneous on $\mathcal{S}$.

Definition $2.2([2])$. A linear functional $\mathrm{L}: \mathcal{S} \rightarrow \mathbb{R}$ satisfying $\mathrm{L}(K+J)$ $\mathrm{L}(K)+\mathrm{L}(J), \mathrm{L}(\lambda K)=\lambda \mathrm{L}(K)$ for $K, J \in \mathcal{S}, \lambda \in \mathbb{R}$, is said to be positive if $\mathrm{L}(K)>0$ whenever $K \in \mathcal{S}$ and $K>0$.

Lemma 2.1. Let $A, B \in \mathbb{R}^{n \times n}$ be real symmetric matrices. Then

$$
\operatorname{tr}\left[(A+B)^{2}\right] \leq 2\left(\operatorname{tr} A^{2}+\operatorname{tr} B^{2}\right) .
$$

Lemma 2.1 follows from the monontonicity of the operator tr and the inequality $(A+B)^{*} P(A+B) \leq 2\left(A^{*} P A+B^{*} P B\right)$ for $P^{*}=P>0$.

In all the following theorems, we will assume that $r, P, Q$ satisfy the assumptions stated right after (1), that the function $H(t, s)$ satisfies (H1)(H3) and that $A_{\tau}^{\varrho}$ is defined by (2) for some $\varrho \in C^{1}\left(\left[t_{0}, \infty\right),(0, \infty)\right)$ and $\tau \geq t_{0}$.

THEOREM 2.1. Suppose there exists a monotone subhomogeneous functional $p$ on $\mathcal{S}$ such that $r(t), P(t)$ and $Q(t)$ satisfy

$$
\limsup _{t \rightarrow \infty} H^{-1}\left(t, t_{0}\right) p\left[A_{t_{0}}^{\varrho}\left(Q-\left\{r+\lambda-\varrho^{\prime} \varrho^{-1}\right\}^{2} P / 4\right)\right]=\infty .
$$

Then the system (1) is oscillatory.

Proof. Suppose to the contrary that there exists a nonoscillatory prepared solution $Y(t)$ of (1). Without loss of generality, we may assume that $\operatorname{det} Y(t) \neq 0$ for $t \geq t_{0}$. Hence the matrix function

$$
W(t)=\left[P(t) Y^{\prime}(t)\right] Y^{-1}(t)
$$

exists on $\left[t_{0}, \infty\right)$. It is easy to see that $W^{*}(t)=W(t)$ and $W(t)$ satisfies the Riccati equation

$$
W^{\prime}(t)=-Q(t)-r(t) W(t)-W(t) P^{-1}(t) W(t)
$$

for $t \in\left[t_{0}, \infty\right)$. By (P4) and (H3),

$$
A_{\tau}^{\varrho}\left(W^{\prime}\right)=-H(\tau, t) W(\tau) \varrho(\tau)+A_{\tau}^{\varrho}\left(\left[\lambda-\varrho^{-1} \varrho^{\prime}\right] W\right),
$$

and by (5), $W^{\prime}=-Q-r W-W P^{-1} W$. From the formulae above we get

$$
-H W \varrho=A_{\tau}^{\varrho}\left(W P^{-1} W+2 \alpha W+Q\right), \quad \text { where } \quad \alpha=\frac{1}{2}\left(\lambda+r-\varrho^{-1} \varrho^{\prime}\right) .
$$


Applying to the last formula the identity

$$
W P^{-1} W+2 \alpha W+Q=\left(P^{-1} W+\alpha I\right)^{*} P\left(P^{-1} W+\alpha I\right)+Q-\alpha^{2} P
$$

one obtains

$$
\begin{aligned}
& H(t, \tau) \varrho(\tau) W(\tau) \\
& \quad=A_{\tau}^{\varrho}\left(\left(P^{-1} W+\alpha I\right)^{*} P\left(P^{-1} W+\alpha I\right)\right)+A_{\tau}^{\varrho}\left(Q-\alpha^{2} P\right),
\end{aligned}
$$

from which, since $P>0$, it follows that

$$
A_{\tau}^{\varrho}\left(Q-\alpha^{2} P\right) \leq H(t, \tau) \varrho(\tau) W(\tau)
$$

and, as a consequence,

$$
p\left[A_{\tau}^{\varrho}\left\{Q-\left\{r+\lambda-\varrho^{\prime} \varrho^{-1}\right\}^{2} P / 4\right\}\right] \leq H(t, \tau) \varrho(\tau) p[W(\tau)] .
$$

Set $\tau=t_{0}$ in (8) and divide the resulting inequality by $H\left(t, t_{0}\right)$ to obtain

$$
\left[H\left(t, t_{0}\right)\right]^{-1} p\left[A_{t_{0}}^{\varrho}\left\{Q-\left\{r+\lambda-\varrho^{\prime} \varrho^{-1}\right\}^{2} P / 4\right\}\right] \leq \varrho\left(t_{0}\right) p\left[W\left(t_{0}\right)\right],
$$

a contradiction with (3). This completes the proof.

Note that the result of Erbe et al. stated in the first section does not require $H(t, s)$ to satisfy conditions $(\mathrm{H} 2)$ and (H3). But they considered a special case of $(1): r(t) \equiv 0$.

Theorem 2.2. Suppose that $\varrho \in C^{1}\left(\left[t_{0}, \infty\right),(0, \infty)\right), H(t, s)$ and $\lambda(t, s)$ are continuous on $D=\left\{(t, s) \mid t \geq s \geq t_{0}\right\}$ such that strict inequality holds in (H1). Suppose further that $\partial H(t, s) / \partial s$ is nonpositive and continuous for $t \geq s \geq t_{0}$, and

$$
0 \leq-\partial H(t, s) / \partial s=\lambda(t, s) H^{1 / 2}(t, s), \quad(t, s) \in D .
$$

If there exists a monotone subhomogeneous functional $p$ on $\mathcal{S}$ such that

$$
\limsup _{t \rightarrow \infty} H^{-1}\left(t, t_{0}\right) p\left[A_{t_{0}}^{\varrho}\left(Q-\left\{r+\lambda H^{-1 / 2}-\varrho^{\prime} \varrho^{-1}\right\}^{2} P / 4\right)\right]=\infty,
$$

then the system (1) is oscillatory.

Indeed, since $H(t, s)>0$, condition (10) is equivalent to (H3) with $\lambda_{1}(t, s)=-\lambda(t, s) / H(t, s)$. Theorem 2.2 is thus a corollary of Theorem 2.1.

REMARK 2.1. Theorems 2.1 and 2.2 remain valid if a monotone subhomogeneous functional $p: \mathcal{S} \rightarrow \mathbb{R}$ is replaced by a linear positive functional $\mathrm{L}: \mathcal{S} \rightarrow \mathbb{R}$. In fact, it is easy to see that if $\mathrm{L}$ is linear and positive, then it is monotone subhomogeneous.

3. Oscillation in terms of positive linear functionals. Let $\varrho(s)=$ $\exp \left\{\int_{t_{0}}^{s} r(\tau) d \tau\right\}$ and $P(t)=I$ in (3). Then Theorem 2.1 and Remark 2.1 yield 
Corollary 3.1. Let $\alpha>1$. Suppose that $r$ and $Q$ satisfy

and

$$
\limsup _{t \rightarrow \infty} \frac{1}{t^{\alpha}} \mathrm{L}\left[\int_{t_{0}}^{t}(t-s)^{\alpha} Q(s) \exp \left(\int_{t_{0}}^{s} r(\tau) d \tau\right) d s\right]=\infty
$$

$$
\lim _{t \rightarrow \infty} \frac{1}{t^{\alpha}} \int_{t_{0}}^{t}(t-s)^{\alpha-2} \exp \left(\int_{t_{0}}^{s} r(\tau) d \tau\right) d s<\infty .
$$

Then the system (1) with $P(t) \equiv I$ is oscillatory.

In particular, if we choose $\mathrm{L}[K]=\operatorname{tr}[K]$, we get the following theorem.

Theorem 3.1. Assume that $P^{-1}(t) \geq I_{n}$. Suppose there are continuous functions $\phi_{1}, \phi_{2}$ on $\left[t_{0}, \infty\right)$ such that

$$
\begin{gathered}
\limsup _{t \rightarrow \infty} H^{-1}\left(t, t_{0}\right) \operatorname{tr}\left[A_{\tau}^{\varrho}(Q)\right] \geq \phi_{2}(\tau), \quad \tau \geq t_{0}, \\
\lim _{t \rightarrow \infty} H^{-1}\left(t, t_{0}\right) \operatorname{tr}\left(A_{\tau}^{\varrho}\left\{\left[r+\lambda-\varrho^{\prime} \varrho^{-1}\right]^{2} P\right\}\right) \leq \phi_{1}(\tau), \quad \tau \geq t_{0},
\end{gathered}
$$

and

$$
\lim _{t \rightarrow \infty} H^{-1}\left(t, t_{0}\right) A_{\tau}^{\varrho}\left\{\varrho^{-2}\left[\phi_{2}-\phi_{1} / 4\right]_{+}^{2}\right\}=\infty, \quad \tau \geq t_{0},
$$

where $\phi_{+}(t)=\max \{\phi(t), 0\}$. Then the system (1) is oscillatory.

Proof. Suppose to the contrary that there exists a nonoscillatory prepared solution $Y(t)$ of (1). Without loss of generality, we may assume that $\operatorname{det} Y(t) \neq 0$ for $t \geq t_{0}$. Define $W(t)$ by (4). As in the proof of Theorem 2.1, we deduce that (7) implies

$$
(H(t, \tau) / H(t, s)) \varrho(\tau) W(\tau) \leq(1 / H(t, s)) A_{\tau}^{\varrho}\left(Q-\alpha^{2} P\right) .
$$

Hence by $(\mathrm{H} 2),(12)$ and $(13)$ we get $K_{0} \varrho(\tau) \operatorname{tr}[W(\tau)] \leq \phi_{2}(\tau)-(1 / 4) \phi_{1}(\tau)$ and, consequently, taking traces of both sides and passing with $t$ to infinity,

$$
\lim _{t \rightarrow \infty} \frac{1}{H\left(t, t_{0}\right)} \operatorname{tr}\left[A_{t_{0}}^{\varrho}\left(W^{2}(t)\right)\right]=\infty .
$$

Setting in (6) $\tau=t_{0}$ and defining $G=\left(P^{-1} W+\alpha I\right)^{*} P\left(P^{-1} W+\alpha I\right)$, we obtain $W\left(t_{0}\right) \varrho\left(t_{0}\right) \leq\left(1 / H\left(t, t_{0}\right)\right) A_{\tau}^{\varrho}\left(G+\left(Q-\alpha^{2} P\right)\right)$. By (12) and (13),

$$
\begin{aligned}
\lim _{t \rightarrow \infty}\left(1 / H\left(t, t_{0}\right)\right) A_{t_{0}}^{\varrho}(G) & =W\left(t_{0}\right) \varrho\left(t_{0}\right)-\lim _{t \rightarrow \infty}\left(1 / H\left(t, t_{0}\right)\right) A_{t_{0}}^{\varrho}\left(Q-\alpha^{2} P\right) \\
& \leq W\left(t_{0}\right) \varrho\left(t_{0}\right)+\phi_{2}\left(t_{0}\right)-\phi_{1}\left(t_{0}\right)<\infty .
\end{aligned}
$$

Now $W^{2} \leq W^{*} P^{-1} W$ because $P^{-1} \geq I$. But

$$
\begin{aligned}
W^{*} P^{-1} W & =(W+\alpha P-\alpha P)^{*} P^{-1}(W+\alpha P-\alpha P) \\
& =\left(P^{-1} W+\alpha P\right)^{*} P^{-1}\left(P^{-1} W+\alpha P\right) .
\end{aligned}
$$

By Lemma 1.1, we conclude that $W^{2} \leq 2\left(G+\alpha^{2} P\right)$, so

$$
\operatorname{tr}\left[A_{t_{0}}^{\varrho}\left(W^{2}\right)\right] \leq 2 \operatorname{tr}\left[A_{t_{0}}^{\varrho}(G)\right]+2 \operatorname{tr}\left[A_{t_{0}}^{\varrho}\left(\alpha^{2} P\right)\right],
$$


which by (16) and assumption (13) implies that

$$
\lim _{t \rightarrow \infty} \frac{1}{H\left(t, t_{0}\right)} \operatorname{tr}\left[A_{t_{0}}^{\varrho}\left(W^{2}(t)\right)\right]<\infty,
$$

contradicting (15). The proof is complete.

In case $\mathrm{L}[A]=\operatorname{tr}[A]$, we obtain some interesting results. By arguments similar to those presented in Wong [3], we may show the following corollaries:

Corollary 3.2. Let $\alpha>1$. Suppose that $r \in C^{1}\left[t_{0}, \infty\right)$ and $Q(t)$ satisfy

$$
\limsup _{t \rightarrow \infty} \frac{1}{t^{\alpha}} \operatorname{tr}\left(\int_{t_{0}}^{t}(t-s)^{\alpha}\left\{Q-\frac{r^{2}}{4} I_{n}-\frac{r^{\prime}}{2} I_{n}\right\} d s\right)=\infty .
$$

Then the system (1) with $P(t) \equiv I$ is oscillatory.

Corollary 3.3. Let $\alpha>1$. Suppose that $\varrho \in C^{1}\left[t_{0}, \infty\right), \varrho(t)>0$,

$$
\int_{t_{0}}^{\infty} \frac{\varrho^{\prime}(s)^{2}}{\varrho(s)} d s=M_{0}<\infty, \quad \lim _{t \rightarrow \infty} \int_{t_{0}}^{t} r^{2}(s) \varrho(s) d s<\infty,
$$

and

$$
\lim _{t \rightarrow \infty} \frac{1}{t^{\alpha}} \operatorname{tr}\left(\int_{t_{0}}^{t}(t-s)^{\alpha} Q(s) \varrho(s) d s\right)=\infty .
$$

Then the system (1) with $P(t) \equiv I$ is oscillatory.

In case $\varrho(s)=\exp \left\{\int_{t_{0}}^{s} r(\tau) d \tau\right\}$ in (3), Theorem 2.1 and Remark 2.1 yield

Corollary 3.4. Let $\alpha>1$. Suppose that $r$ and $Q$ satisfy

$$
\begin{gathered}
\limsup _{t \rightarrow \infty} \frac{1}{t^{\alpha}} \operatorname{tr}\left(\int_{t_{0}}^{t}(t-s)^{\alpha} Q(s) \exp \left(\int_{t_{0}}^{s} r(\tau) d \tau\right) d s\right)=\infty, \\
\lim _{t \rightarrow \infty} \frac{1}{t^{\alpha}} \int_{t_{0}}^{t}(t-s)^{\alpha-2} \exp \left(\int_{t_{0}}^{s} r(\tau) d \tau\right) d s<\infty
\end{gathered}
$$

Then the system (1) with $P(t) \equiv I$ is oscillatory.

EXAmple 3.1. Consider the system (1) where

$$
P(t)=I_{2}, \quad r(t)=\cos t, \quad Q(t)=\operatorname{diag}[\sin t,-\sin t], \quad t \geq 0 .
$$

Note that both $r$ and $Q$ are oscillatory, that is, they take on alternating signs for large values of $t$. Let us apply Corollary 3.1 to our equation by defining $\mathrm{L}[R]=r_{11}$ where $R=\left(r_{i j}\right)$. It is easy to see 


$$
\begin{aligned}
\frac{1}{t^{\alpha}} \mathrm{L}\left[\int_{t_{0}}^{t}(t-s)^{\alpha} Q(s) \exp \right. & \left.\left(\int_{t_{0}}^{s} r(\tau) d \tau\right) d s\right] \\
& =\frac{1}{t^{\alpha}} \int_{t_{0}}^{t}(t-s)^{\alpha}(\sin s) \exp \left(\int_{t_{0}}^{s} \cos \tau d \tau\right) d s
\end{aligned}
$$

Note further that

$$
\lim _{t \rightarrow \infty} t^{-\alpha} \int_{0}^{t}(t-s)^{\alpha-2} \exp (\sin s) d s=0
$$

By arguments similar to those presented in Wong [3], the integral in (21) diverges as $t \rightarrow \infty$. By Corollary 3.1, we see that every prepared solution of our system (1) defined by (20) is oscillatory.

However, if we choose $\mathrm{L}[R]=\operatorname{tr} R$, then $\operatorname{tr} Q(t)=0$. In this case, our system is still oscillatory. Note that our system can be written as a pair of scalar equations:

$$
\begin{aligned}
x^{\prime \prime}+(\cos t) x^{\prime}+(\sin t) x & =0, \\
y^{\prime \prime}+(\cos t) y^{\prime}-(\sin t) y & =0 .
\end{aligned}
$$

From the above discussion, it is easy to see (23) is oscillatory. But the system (24) has a nonoscillatory solution $x(t)=\exp \{-\sin t\}$. Hence, Theorems 2.1, 2.2 and Theorem 3.2 cannot be applied to our system, neither can the oscillation criteria in [1-4].

EXample 3.2. Consider the $n$-dimensional system (1) where

$$
P(t)=I_{n}, \quad r(t)=\cos t, \quad Q(t)=I_{n} \sin t, \quad t \geq 0 .
$$

Again, both $r$ and $Q$ are allowed to be oscillatory. By arguments similar to those above, we can obtain (22) and

$$
\limsup _{t \rightarrow \infty} t^{-\alpha} \operatorname{tr}\left(\int_{t_{0}}^{t}(t-s)^{\alpha} Q(s) \exp \left(\int_{t_{0}}^{s} r(\tau) d \tau\right) d s\right)=\infty .
$$

But (22) and (26) imply conditions in Corollary 3.4, proving that system (1) defined by (25) is oscillatory. However, the oscillation of our system cannot be demonstrated by the criteria in [1-4].

Acknowledgments. The authors are greatly indebted to the referee for his valuable comments that have led to the present version of this paper.

\section{References}

[1] L. H. Erbe, Q. Kong and S. Ruan, Kamenev type theorems for second order matrix differential systems, Proc. Amer. Math. Soc. 117 (1993), 957-962.

[2] P. Hartman, Oscillation criteria for selfadjoint second-order differential systems and "principal sectional curvatures", J. Differential Eqations 34 (1979), 326-338. 
[3] J. S. W. Wong, On Kamenev-type oscillation criteria for second order differential equations with damping, J. Math. Anal. Appl. 258 (2001), 244-257.

[4] Q. G. Yang, R. Mathsen and S. M. Zhu, Oscillation theorems for self-adjoint matrix Hamiltonian systems, J. Differential Eqations 190 (2003), 306-329.

Department of Mathematics

South China University of Technology

Guangzhou, Guangdong 510640, P.R. China

E-mail: yangqigui@263.net
Department of Mathematics

Tsinghua University Hsinchu, Taiwan 30043, R.O. China

E-mail: sscheng@math.nthu.edu.tw

Reçu par la Rédaction le 18.10.2004

Révisé le 22.2.2005 